\title{
GaN Transistors for Miniaturized Pulsed-Power Sources
}

\author{
Mohammad Samizadeh Nikoo, Member, IEEE, Armin Jafari, and Elison Matioli, Member, IEEE
}

\begin{abstract}
In this paper, we discuss and demonstrate the potential of normally-ON GaN high-electron-mobility transistors (HEMTs) as opening switches in miniaturized pulsed-power circuits. The high breakdown electric field of $\mathrm{GaN}(\sim 3 \mathrm{MV} / \mathrm{cm})$ makes it possible to fabricate high-voltage devices in small dimensions, resulting in smaller parasitics, and faster switching times. GaN HEMTs as opening switches are compatible with inductive topologies, which offer more than one order of magnitude higher energy density than capacitive topologies, allowing a further miniaturization of pulsed-power systems. In this work, we demonstrate the application of fabricated $1.5 \mathrm{kV}$ normally-on GaN HEMTs on an inductive switching topology, resulting in 300x-fold voltage step-up, which makes it possible to generate high-voltage pulses with a low-voltage DC source in miniaturized circuits.
\end{abstract}

Index Terms-Pulsed-power, fast switching, high electron mobility transistor (HEMT), GaN, opening switch, high energy density.

\section{INTRODUCTION}

$\mathrm{C}$ ONVERSION of low-power long pulses into highamplitude short pulses, called pulse compression, is a general idea behind any kind of pulsed-power systems. Such high-power pulses have opened promising pathways to address some of the most important challenges of the current century, such as conversion of greenhouse gases like $\mathrm{CO}_{2}$ and $\mathrm{CH}_{4}$ to value added products [1] and cancer treatment [2].

For instance, a high-voltage pulse generator is used to drive a plasma device that produces energetic electrons in a greenhouse gas environment. Through collisions, the electron energy is transferred to different channels of excitation, ionization, resulting in the dissociation of the gas molecules [3]. The effect of pulsed electric field (PEF) generated by high-voltage pulses on cell membrane permeabilization is the origin of many studies in this field [4]. The rise time, period and amplitude of the electric pulses result in different effects on cells. Microsecond PEFs ( $\mu \mathrm{SPEFs}$ ) can permeabilize the external membrane of cells, while permeabilization of internal membranes requires nanosecond PEFs (nsPEFs) [5], [6].

The application of PEF can also improve the uptake of antitumor drugs by permeabilized cell membrane [7], [8].

Manuscript received December 13, 2018.

The authors are with the Power and Wide-band-gap Electronics Research Laboratory (POWERlab), École polytechnique fédérale de Lausanne (EPFL), CH-1015 Lausanne, Switzerland (e-mail: mohammad.samizadeh@epfl.ch, elison.matioli@epfl.ch).

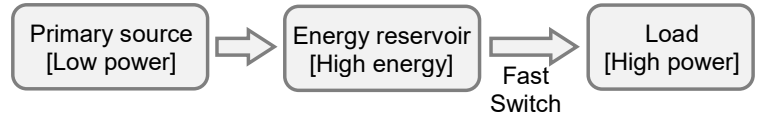

Fig. 1. A general block diagram for a pulsed-power system.

Increasing the intensity of the PEFs causes irreversible electroporation (IRE) and necrosis of tumor cells [7], [9], without inducing thermal effect nor requiring extra drugs [10], [11]. Miniaturized pulse generators allow a local generation of high-voltage pulses which are highly desirable to these applications.

Fig. 1 illustrates a general block diagram of a pulsed-power system. A low-power source charges an energy reservoir, which can be a capacitor, an inductor, or a cavity, and the stored energy is discharged into the load using a fast switch, which is a key element in a pulsed-power system. Depending on the employed energy reservoir, a closing or an opening switch is needed. Some devices like gas switches [12] and fast ionizing dynistors (FIDs) [13] can just perform as closing switch, while other devices like semiconductor opening switches (SOSs) [14], [15] and drift step recovery diodes (DSRDs) [16], [17] perform as opening switch (some switches can act in both regimes). An important fact is that the energy density in inductive-based pulsed-power sources (based on opening switches) is typically more than one order of magnitude larger that in capacitive-based pulsed-power sources (based on closing switches) [18]. Therefore, opening switches offer an increased energy density allowing the miniaturization of pulsed-power systems, which is the goal of this paper.

Semiconductor opening switch (SOS) and drift step recovery diode (DSRD) are two-port opening switches specifically used in pulsed-power systems. In SOS the current interruption occurs due to the drastic increase in the resistance of the low-doped part of the p-layer, where the reverse current density exceeds the saturation value [19]. This phenomenon is typically observed for very high forward and reverse current densities. This issue limits the performance of SOS to highcurrent systems. DSRD is also a solid-state device which provides fast switching based on the reverse recovery of a $p-n$ junction [20]. Pulses with fast rise times can be generated by these switches [21]. The power capability, fast rise-time and high repetition rate of these two-port switches generally cannot be obtained by conventional three-port switches however, two-port switches cannot be triggered by a control signal. Another shortcoming is that these switches are not commercially available, although the basic concept is observable in commercial diodes [22]. 
Conventional semiconductor switches like MOSFETs and IGBTs are not commonly used in pulsed-power systems. As discussed in [23], the large conduction resistance of power MOSFETs and the limited $d i / d t$ of IGBTs are considerable shortcoming that prevent them from being applied to pulsedpower applications. Among power semiconductor devices, the thyristor has a relatively long history in pulsed-power. However, the slow response time of thyristors limits their operation frequency to about $100 \mathrm{~Hz}$ or lower [24].

In this work we investigate the application of $\mathrm{GaN}$ highelectron mobility transistors (HEMTs) in pulsed-power applications for miniaturized pulsed-power systems. $\mathrm{GaN}$ is an interesting material when both high-power and fast dynamics are required. Although the application of $\mathrm{GaN}$ for high-power RF applications has been demonstrated [25], little work has been done on the use of GaN in pulsed-power [26]. The peak drift velocity of $3.1 \times 10^{7} \mathrm{~cm} / \mathrm{s}$ together with the blocking field of $\sim 3 \mathrm{MV} / \mathrm{cm}$ are the highest numbers among all semiconductor candidates $(\mathrm{Si}, \mathrm{GaAs}, 4 \mathrm{H}-\mathrm{SiC})$, resulting in high switching power. The high-power density of $\mathrm{GaN}$ allows a reduction of the size of the switch and the miniaturization of pulsed-power systems. In the second section of the paper, we discuss and model a pulsed-power generator based on GaN HEMT as the opening switch. The fabrication process and characterization of the devices with large breakdown field (higher than in commercially available $\mathrm{GaN}$ devices) are presented in the third section. The switching performance of the devices in a very high step-up miniaturized pulse generator is evaluated in the fourth section.

\section{Methodology}

Fig. 2a shows a simple structure of a pulsed-power generator based on inductive energy storage. The transistor is normally $\mathrm{ON}$ and for $v_{\mathrm{G}}=0$, the current $i_{\mathrm{D}}=I_{0}$ passes through the low on-resistance $\left(R_{\mathrm{ON}}\right)$ of the $\mathrm{GaN}$ transistor (Fig. 2b). The low-power DC source $\left(V_{\mathrm{DD}}\right)$ charges the inductor with a current

$$
I_{0}=\frac{V_{\mathrm{DD}}}{R_{\mathrm{S}}+R_{\mathrm{ON}}}
$$

where $R_{\mathrm{S}}$ is the series resistance of the inductor. In this case, even for small values of $V_{\mathrm{DD}}$, the small $R_{\mathrm{S}}$ and ON resistance of the HEMT, lead to relatively high currents, storing a significant energy into the magnetic field of the inductor

$$
E=\frac{1}{2} L I_{0}^{2} \text {. }
$$

The DC source power charging the inductor is

$$
P_{\text {in }}=\frac{V_{\mathrm{DD}}^{2}}{R_{\mathrm{S}}+R_{\mathrm{ON}}} \text {. }
$$

After charging the inductor, a gate control signal is applied to switch OFF the transistor. Since there is no abrupt change in the current of the inductor, the huge reduction in the drain current leads to a huge increase in the current of load, resulting in a high voltage output pulse. Ideally, if the switch
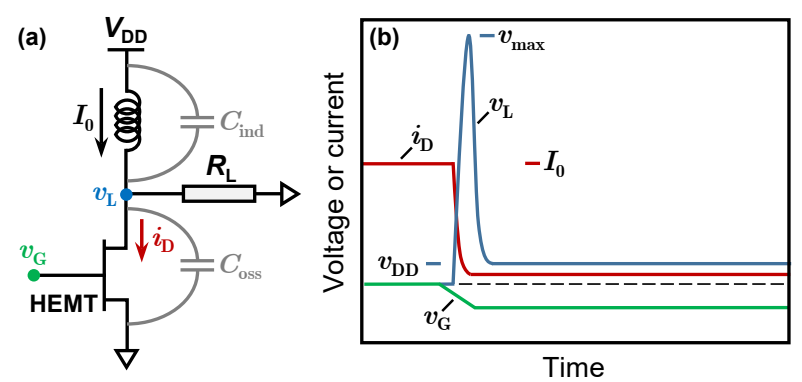

Fig. 2. (a) A simple structure of an inductive energy storage pulsed-power generator based on a HEMT switch. (b) Key waveforms of the considered pulsed-power generator.

blocked the drain current in zero time, the output peak voltage could be approximated by [20]

$$
v_{\max }=\left(1.18 R_{L} \| Z\right) I_{0}
$$

Where

$$
Z=\sqrt{L / C}
$$

and $C=C_{\text {oss }}+C_{\text {ind }}$ is the sum of the effective parasitic capacitance of the HEMT and inductor, respectively. Assuming $R_{\mathrm{L}}$ and $Z$ to be much larger than $R_{\mathrm{S}}$, a step-up in voltage with $v_{\max } \gg v_{\text {DD }}$ can be obtained. The output power $P_{\text {out }}$ is also much larger than the input power for well-tuned $R_{\mathrm{L}}$. Here, to have an independent discussion from the load $R_{\mathrm{L}}$, we consider the case of $R_{\mathrm{L}} \gg Z$ and use the step-up ratio $v_{\max } / v_{\mathrm{DD}}$ as benchmark. In many applications, the condition of large loads is applicable, thus high-voltage pulses are achievable. For $R_{\mathrm{L}} \gg Z$ one can rewrite (4) as

$$
v_{\max }=Z I_{0}
$$

which results in a step-up voltage ratio of

$$
G_{v} \triangleq \frac{v_{\mathrm{max}}}{V_{\mathrm{DD}}}=\frac{Z}{R_{\mathrm{S}}+R_{\mathrm{ON}}} .
$$

Based on this analyses, higher step-up ratios can be obtained by increasing current or $Z$. Equation (7) is obtained for an ideal switch with zero switching time, however, it is applicable for fast switches, when the switching time $t_{\mathrm{sw}}$ satisfies

$$
t_{\mathrm{sw}} \ll \sqrt{L C}
$$

In this case, just a tiny part of the energy $E$ is dissipated in the HEMT switch. The output waveform can be expressed as

$$
v_{\text {out }}=v_{\max } \sin \left(\frac{t}{\sqrt{L C}}\right), \quad 0<t<\pi \sqrt{L C}
$$

which results in a pulse with a full-width at half maximum (FWHM) of

$$
\mathrm{FWHM}=\frac{2 \pi}{3} \sqrt{L C}
$$

For further sharpening in the output pulse one can use a selfbreaking spark-gap before the load. In this case, the load $R_{\mathrm{L}}$ does not affect the circuit until the spark-gap turns on [22]. As a result, a high step-up in no-load condition is required.

\section{DEVICE FABRICATION AND CHARACTERIZATION}

To demonstrate the promising performance of GaN-based HEMTs in pulsed-power switching, high-voltage devices with ampere-range current capability were fabricated based on an 
epitaxial structure consisting of AlGaN (19.6 nm) / GaN (420 $\mathrm{nm})$ followed by a $4.2 \mu$ m-thick buffer grown on a 6 -inch silicon substrate. The first fabrication step was the definition of the isolated mesa region by photolithography followed by $500 \mathrm{~nm}$-deep $\mathrm{Cl}_{2}$-based inductively coupled plasma (ICP) etching to reach the highly resistive buffer layer. The second step was the definition of source and drain ohmic contacts, using a metal stack of Ti $(200 \AA) / \mathrm{Al}(1200 \AA) / \mathrm{Ti}(400 \AA) / \mathrm{Ni}$ $(600 \AA) / \mathrm{Au}(500 \AA)$ deposited in the contact regions by electron-beam evaporation and annealed at $780^{\circ} \mathrm{C}$ to form ohmic contacts. The last step of the process was the deposition of $\mathrm{Ni}(300 \AA) / \mathrm{Au}(1500 \AA)$ as the gate electrode and pads, also defined by photolithography.

Fig. 3a and Fig. 3b show the micrograph and SEM image of the fabricated devices, respectively. The area of the mesa was $0.8 \mathrm{~mm}^{2}$, and the gate length and drain-to-gate distance were $2.5 \mu \mathrm{m}$ and $25 \mu \mathrm{m}$, respectively. The device cross section is shown in Fig. 3c. The transfer and output characteristics of the device were separately measured to evaluate their DC performance. The transfer characteristics of three different devices of the same kind are plotted in Fig. 4a to show the consistency of the fabricated devices. As discussed before, the transistors are intrinsically normally ON, which is shown by the negative threshold voltage of $-4 \mathrm{~V}$. Fig. $4 \mathrm{a}$ also shows the low gate leakage current. The output characteristics of the device (Fig. $4 \mathrm{~b}$ ) shows an ON resistance $R_{\mathrm{ON}}$ of $10 \Omega$, which is in the range of series resistance of the inductor. Fabrication of devices with smaller ON resistances is not a requirement in this application, since it would increase the gate capacitance and $C_{\text {oss }}$, which would degrade the switching performance of the device without any considerable gain in charging current $I_{0}$.

The breakdown test on the devices was performed using Keysight B1505A power device analyzer connected to high voltage probe station. As shown in Fig. 5, the devices
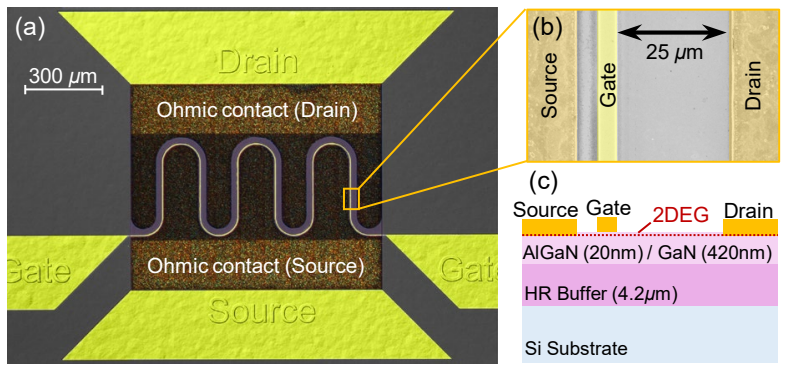

Fig. 3. (a) Micrograph of the fabricated device together with (b) the SEM image, and (c) cross section of the device.
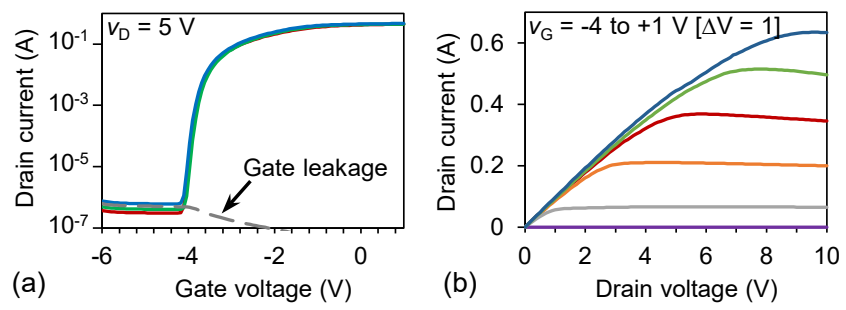

Fig. 4. (a) Transfer characteristics of three different devices measured at the drain voltage of $v_{\mathrm{D}}=5 \mathrm{~V}$ (b) Output characteristics of the fabricated devices for gate voltages between $-4 \mathrm{~V}$ and $1 \mathrm{~V}$ with step of $1 \mathrm{~V}$.
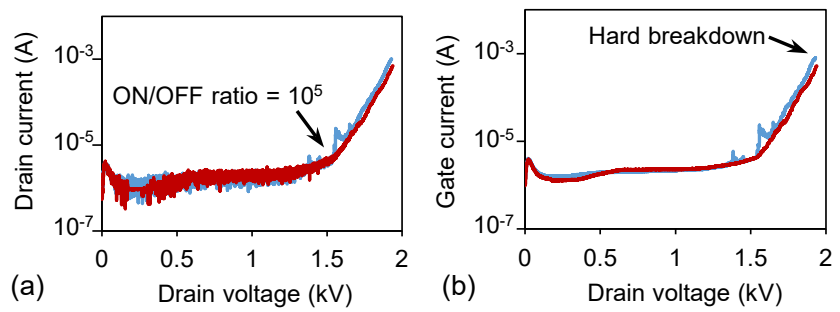

Fig. 5. (a) Drain current and (b) gate current of two fabricated devices versus drain voltage for $v_{\mathrm{G}}=-8 \mathrm{~V}$ showing the stability of the fabrication process. The soft and hard breakdown voltages of $1.5 \mathrm{kV}$ and $2 \mathrm{kV}$, respectively, were obtained.

presented large soft breakdown voltage of $1.5 \mathrm{kV}$ measured at $V_{\mathrm{G}}=-8 \mathrm{~V}$ along with a small gate leakage current. The hard (destructive) breakdown was about $2 \mathrm{kV}$. Higher voltage can be obtained by using different epitaxies, field-plate and larger gate-drain distances [27], [28].

\section{PULSED-POWER SWITCHING}

In order to demonstrate the switching performance of $\mathrm{GaN}$ transistors in the inductive pulsed-power topologies we realized the circuit of Fig. 2a using the fabricated $\mathrm{GaN}$ transistors and $1 \mathrm{mH}$ inductors from Coilcraft. Fig. 6 illustrates the main components of the circuit, showing their small dimensions. The inset of Fig. 6, shows the zoomed-in image of the fabricated device. The impedance of the employed inductor was measured with Keysight E4990A 50 MHz impedance analyzer, as shown in Fig. 7. Based on the measurement, we extracted the parasitic components of the

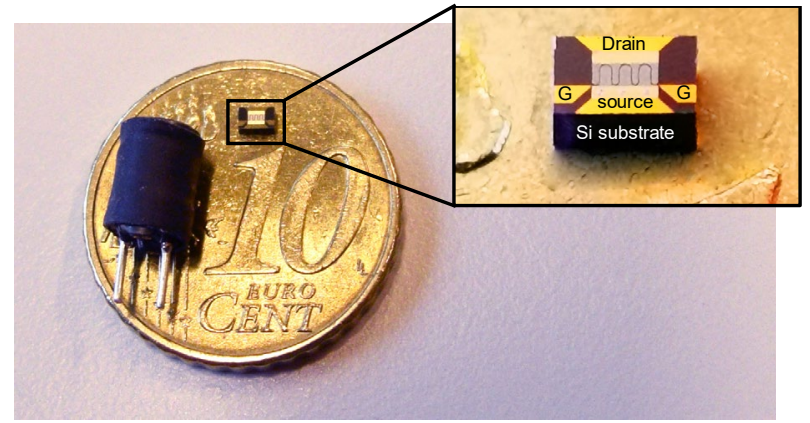

Fig. 6. The main components in the miniaturized pulse generator showing their small dimensions, where the inset shows the zoomed-in image of the fabricated device.

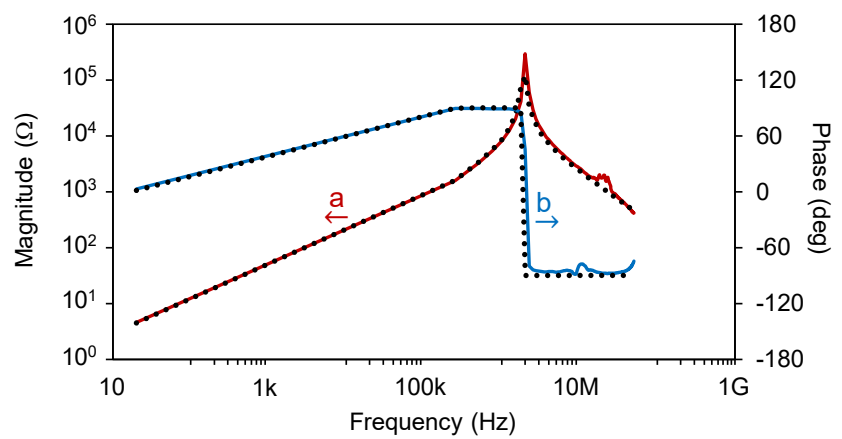

Fig. 7. The measured (solid lines) and modeled (dotted lines) impedance of the employed inductor (a) Magnitude, and (b) Phase. 


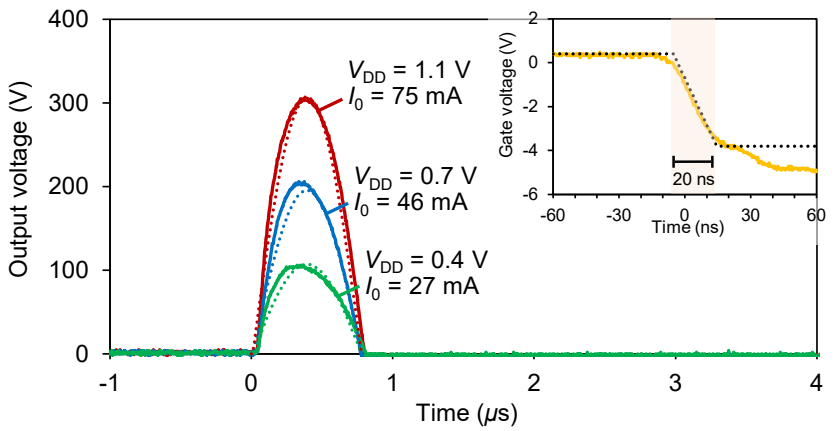

Fig. 8. The measured (solid line) and simulated (dotted line) output waveforms for three different values of $V_{\mathrm{DD}}$ using the fabricated devices in the circuit shown in Fig. 2a with $L=1 \mathrm{mH}$. The inset shows the measured (solid line) and simulated (dotted line) gate voltage signal.
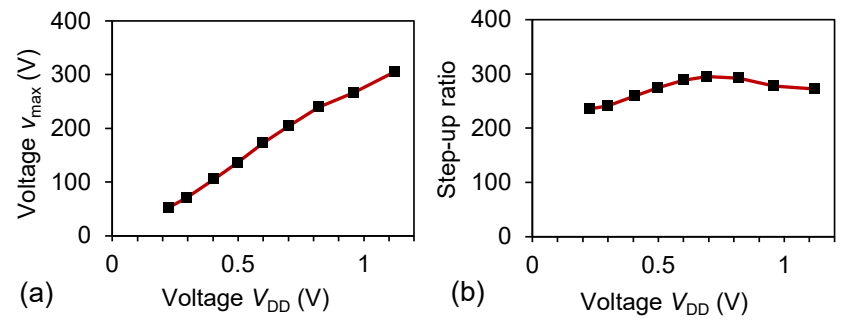

Fig. 9. (a) The measured output maximum voltage, and (b) voltage step-up ratio versus the bias voltage $V_{\mathrm{DD}}$.

inductors, modelled as an ideal $1 \mathrm{mH}$ inductor in series with a $4.5 \Omega$ resistance $\left(R_{\mathrm{S}}\right)$, all in parallel with a $7 \mathrm{pF}$ capacitance $\left(C_{\text {ind }}\right)$. For time-domain measurements we used Tektronix MIDO3104 $1 \mathrm{GHz} 5 \mathrm{GS} / \mathrm{s}$ digital oscilloscope. TPP1000 passive voltage probes were used for the measurements, which added $C_{\text {probe }}$ of $4 \mathrm{pF}$ to the parasitic capacitances at the output node. Adding the HEMT $C_{\text {oss }}$ together with the parasitic capacitance of the output node to $C_{\text {ind }}$ and $C_{\text {probe }}$ resulted in a total capacitance of $60 \mathrm{pF}$. The inductor was charged with a low-voltage DC source and high peak voltage pulses were generated by blocking the current of inductor using the fabricated HEMT as an opening switch. Fig. 8 shows the waveforms generated based on the implemented structure for three different values of $v_{\mathrm{DD}}$, together with the gate signal. A good match between simulation and measurement was obtained. All of the generated pulses have the same FWHM pulse width is $530 \mathrm{~ns}$ which is in agreement with the theoretical prediction in (10)

$$
\mathrm{FWHM}=\frac{2 \pi}{3} \sqrt{(1 \mathrm{mH})(60 \mathrm{pF})}=513 \mathrm{~ns}
$$

Fig. 9a shows the output peak voltage of the generated pulses for different bias levels. The voltage step-up ratio is shown in Fig. 9b revealing a large step-up ratio of about 300 times. Using the proposed model in Fig. 2a, one can theoretically predict the experimental measurement and simulation results. Based on (5), we obtain $Z=4.1 \mathrm{k} \Omega$ which results in the theoretical step-up ratio of $G_{v}=273$ which is in agreement with the measurement results. Such a high level of step-up in a miniaturized circuit opens a promising way for future pulsedpower technology with applications in biology and environmental sciences.

\section{CONCLUSION}

Here we demonstrated the performance of small-scale GaNbased HEMTs for pulsed-power switching. DC characterization of the fabricated devices showed $2 \mathrm{kV}$ hard breakdown voltage together with an ampere-range current capability. The device was employed in an inductiveswitching topology and showed 300 times step-up in the voltage levels. The main goal of the paper was demonstration of pulse generation with very high step-up ratio from a lowvoltage supply in a miniaturized circuit.

A simple model was provided, which matched explained very well the experimental results. This work reveals the enormous potential of GaN fast opening switches to take advantage of the higher energy density of magnetic materials for efficient and miniaturized pulsed-power switches.

\section{REFERENCES}

[1] A. Bogaerts, T. Kozák, K. van Laer, and R. Snoeckx, "Plasma-based conversion of $\mathrm{CO} 2$ : current status and future challenges," Faraday Discuss., vol. 183, pp. 217-232, Jun. 2015.

[2] S. J. Beebe, P. M. Fox, L. J. Rec, K. Somers, R. H. Stark, and K. H. Schoenbach, "Nanosecond pulsed electric field (nsPEF) effects on cells and tissues: Apoptosis induction and tumor growth inhibition," IEEE Trans. Plasma Sci., vol. 30, no. 1, pp. 286-292, Feb. 2002.

[3] R. Snoeckx and B. Annemie, "Plasma technology-a novel solution for $\mathrm{CO}_{2}$ conversion?," Chem. Soc. Rev., vol. 46, no. 19, pp. 5805-5863, 2017.

[4] M. Breton and L. M. Mir, "Microsecond and nanosecond electric pulses in cancer treatments," Bioelectromagnetics, vol. 33, no. 3, pp. 106-123, Aug. 2011.

[5] H. Hanna, A. Denzi, M. Liberti, F. M. André, and L. M. Mir, "Electropermeabilization of inner and outer cell membranes with microsecond pulsed electric fields: quantitative study with calcium ions," Sci. Rep., vol. 7, p. 13079, Oct. 2017.

[6] S. D. Jensen, V. A. Khorokhorina, C. Muratori, A. G. Pakhomov, and O. N. Pakhomova, "Delayed hypersensitivity to nanosecond pulsed electric field in electroporated cells," Sci. Rep., vol. 7, p. 10992, Sep. 2017.

[7] A. Silve, I. Leray, C. Poignard, and L. M. Mir, "Impact of external medium conductivity on cell membrane electropermeabilization by microsecond and nanosecond electric pulses," Sci. Rep., vol. 6, p. 19957, Feb. 2016.

[8] J. Dai, S. Wu, Y. Kong, Z. Chi, L. Si, X. Sheng, C. Cui, J. Fang, J. Zhang, and J. Guo, "Nanosecond pulsed electric fields enhance the antitumour effects of the mtor inhibitor everolimus against melanoma," Sci. Rep., vol. 7, p. 39597, Jan. 2017.

[9] L. Carr, S. M. Bardet, R. C. Burke, D. Arnaud-Cormos, P. Leveque, and R. P. O'Connor, "Calcium-independent disruption of microtubule dynamics by nanosecond pulsed electric fields in U87 human glioblastoma cells," Sci. Rep., vol. 7, p. 41267, Jan. 2017.

[10] Ling He, Deyou Xiao, Jianguo Feng, Chenguo Yao, and Liling Tang, "Induction of apoptosis of liver cancer cells by nanosecond pulsed electric fields (nsPEFs)," Med. Oncol., vol. 34, no. 2, p. 24, Jan. 2017.

[11] E. C. Gianulis, C. Labib, G. Saulis, V. Novickij, O. N. Pakhomova, and A. G. Pakhomov, "Selective susceptibility to nanosecond pulsed electric field (nsPEF) across different human cell types," Cell. Mol. Life Sci., vol. 74, no. 9, pp. 1741-1754, May. 2017.

[12] G. J. J. Winandsa, Z. Liu, A. J. M. Pemen, E. J. M. van Heesch, and K. Yan, "Long lifetime, triggered, spark-gap switch for repetitive pulsed power applications," Rev. Sci. Instrum., vol. 76, no. 8, p. 085107, Aug. 2005.

[13] I. V. Grekhov, S. V. Korotkov, A. L. Stepaniants, D.V. Khristyuk, V. B. Voronkov, and Y. V. Aristov, "High-power semiconductor-based nano and subnanosecond pulse Generator with a low delay time," IEEE Trans. Plasma Sci., vol. 33, no. 4, pp. 1240-1244, Aug. 2005.

[14] A. B. J. M. Driessen et al. "Compact pulse topology for adjustable highvoltage pulse generation using an SOS diode," IEEE Trans. Plasma Sci., vol. 42, no. 10, pp. 3083-3088, Oct. 2014.

(C) 2019 IEEE. Personal use of this material is permitted. Permission from IEEE must be obtained for all other uses, in any current or future media, including reprinting/republishing this material for advertising or promotional purposes, creating new collective works, for resale or redistribution to servers or lists, or reuse of any copyrighted component of this work in other works. 
[15] S. N. Rukin et al., "SOS-based pulsed power: Development and applications," in Proc. 12th IEEE Int Pulsed Power Conf., 1999, pp. $153-156$

[16] M. S. Nikoo and S. M-.A. Hashemi, "A compact MW-class short pulse generator," IEEE Trans. Plasma Sci., vol. 46, no. 6, pp. 2059-2063, Jun. 2018.

[17] M. S. Nikoo, S. M.-A. Hashemi, and F. Farzaneh, "A two-stage DSRDbased high-power nanosecond pulse generator," IEEE Trans. Plasma Sci., vol. 46, no. 2, pp. 427-433, Feb. 2018.

[18] J. Mankowski and M. Kristiansen, "A review of short pulse generator technology," IEEE Trans. Plasma Sci., vol. 28, no. 1, pp. 102-108, Oct. 2000.

[19] I. V. Grekhov and G. A. Mesyats, "Physical basis for high-power semiconductor nanosecond opening switches," IEEE Trans. Plasma Sci., vol. 28 , no. 5, pp. 1540-1544, Oct. 2000.

[20] M. S. Nikoo and S. M. A. Hashemi, "High-power nanosecond pulse generator with high-voltage SRD and GDT switch," IEEE Trans. Plasma Sci., vol. 43, no. 9, pp. 3268-3276, Sep. 2015.

[21] L. M. Merensky, et al., "A low-jitter 1.8-kV 100-ps rise-time $50-\mathrm{kHz}$ repetition-rate pulsed-power generator," IEEE Trans. Plasma Sci., vol. 37, no. 9, pp. 1855-1862, Sep. 2009.

[22] M. S. Nikoo, S. M.-A. Hashemi, and M. O. Dilmaghanian, "DSRDbased high-power repetitive short-pulse generator containing GDT: Theory and experiment," IEEE Trans. Plasma Sci., vol. 45, no. 8, pp. 2341-2350, Aug. 2017.

[23] W. Chen et al., "Design and characterization of high di/dt CS-MCT for pulse power applications," IEEE Trans. Electron Devices, vol. 64, no. 10, pp. 4206-4212, Oct. 2017.

[24] W. Jiang et al., "Compact solid-State switched pulsed power and its applications," Proc. IEEE, vol. 92, no. 7, pp. 1180-1196, Jul. 2004.

[25] K. Shinohara et al., "Scaling of GaN HEMTs and schottky diodes for submillimeter-wave MMIC applications," IEEE Trans. Electron Devices, vol. 60, no. 10, pp. 2982-2996, Oct. 2013.

[26] O. S. F. Zucker, P. K. L. Yu, and Y.-M. Sheu, "GaN switches in pulsed power: a comparative study," IEEE Trans. Plasma Sci., vol. 42, no. 5, pp. 1295-1303, May 2014.

[27] Y. Dora, A. Chakraborty, L. McCarthy, S. Keller, S. P. DenBaars, and U. K. Mishra, "High breakdown voltage achieved on $\mathrm{AlGaN} / \mathrm{GaN}$ HEMTs with integrated slant field plates," IEEE Electron Device Lett., vol. 27, no. 9, pp. 713-715, Sep. 2006

[28] H.-S. Lee et al., "3000-V 4.3-m·cm2 InAlN/GaN MOSHEMTs with AlGaN back barrier," IEEE Electron Device Lett., vol. 33, no. 7, pp. 982-984, Jul. 2012.

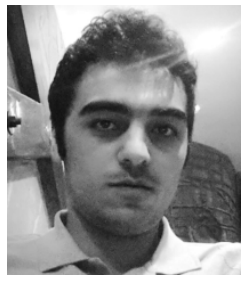

Mohammad Samizadeh Nikoo (M'16) was born in Tehran, Iran, in 1992. He received the B.Sc. and M.Sc. degrees in electrical engineering from the Sharif University of Technology, Tehran, in 2015 and 2017, respectively. He is currently pursuing the Ph.D. degree at the École Polytechnique fédérale de Lausanne, Lausanne, Switzerland. His current research interests include electron devices and ultrafast electronics.

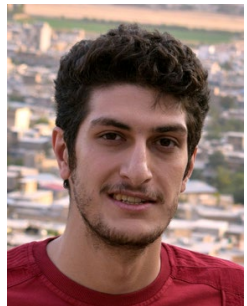

Armin Jafari studied Electrical Engineering at University of Tehran, Tehran, Iran and received his B.Sc. degree in Power in 2016. He is currently pursuing a Ph.D. degree at École Polytechnique fédérale de Lausanne (EPFL), Lausanne, Switzerland. His current research interests include step-up DC-DC converters, power magnetics at high-frequency, wideband-gap device characterisation and measurement of loss in power converters based on heat transfer.

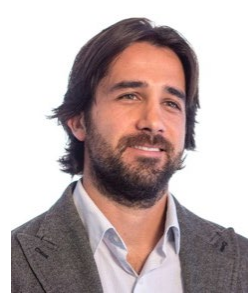

Elison Matioli is an assistant professor in the institute of electrical engineering at Ecole Polytechnique Fédérale de Lausanne (EPFL). He received a B.Sc. degree in applied physics and applied mathematics from Ecole Polytechnique (Palaiseau, France) in 2006 and a Ph.D. degree from the Materials Department at the University of California, Santa Barbara (UCSB) in 2010. He was a post-doctoral fellow in the EECS department at the Massachusetts Institute of Technology (MIT) until 2014. He has received the UCSB Outstanding Graduate Student - Scientific Achievement Award for his Ph.D. thesis, the IEEE George Smith Award, the SNSF Assistant Professor Energy Grant Award, and the ERC Starting Grant Award. 